\title{
Viabilidad de Mezclas Supercríticas en Ciclos Brayton Acoplados a Plantas de Energía Solar Concentrada
}

Paul Tafur-Escanta ${ }^{1}$, Luis Coco-Enríquez ${ }^{1}$ y Javier Muñoz-Antón ${ }^{1}$

${ }^{1}$ Universidad Politécnica de Madrid. C/ José Gutiérrez Abascal, 2. 28006, Madrid, España

17pm.tafur@alumnos.upm.es, ${ }^{2}$ luiscocoenriquez@hotmail.com, 3jamunoz@etsii.upm.es

\section{RESUMEN}

En este trabajo se promueve estudiar la viabilidad del uso de mezclas basadas en $\mathrm{CO}_{2}$ supercrítico que pueda conducir a rendimientos térmicos en los ciclos Brayton más altos que los obtenidos con el fluido estándar (s- $\mathrm{CO}_{2}$ puro). Las configuraciones que se recomienda analizar son las de Recompresión y Recompresión con enfriamiento intermedio en el compresor principal que pueden incluir más de un recalentamiento. Estas configuraciones son muy transcendentales debido a que en términos de eficiencia son las que mayores valores presentan dependiendo de las conductancias de los recuperadores de calor pueden alcanzar eficiencias de entre $47 \%$ y $53 \%$, sin embargo, cuando se añaden caídas de presión en los componentes del ciclo las eficiencias disminuyen a valores de entre $44 \%$ y $50 \%$ cuando su fluido de trabajo es s- $\mathrm{CO}_{2}$ puro, además, los costos totales por capacidad neta instalada son los más bajos en comparación con otras configuraciones.

Palabras Clave: $\mathrm{CO}_{2}$ supercrítico, energía solar concentrada, planta termosolar, mezclas binarias 


\section{Introducción}

El mercado mundial de la energía ha ido cambiando progresivamente en los últimos años. Esto se puede explicar con distintos hechos, siendo los más destacados la creciente preocupación por el medio ambiente y el cambio climático, el incremento del coste de los combustibles fósiles y su agotamiento y el desarrollo de nuevas tecnologías renovables cada vez más competitivas. Se puede ver, desde el punto de vista del análisis estadístico, que en el período 2010-2020 se ha producido una gran revolución en las tecnologías renovables. De hecho, ha habido más instalaciones de este tipo que no renovables desde 2013, alcanzando 1/3 de la capacidad instalada total en todo el mundo. [1]

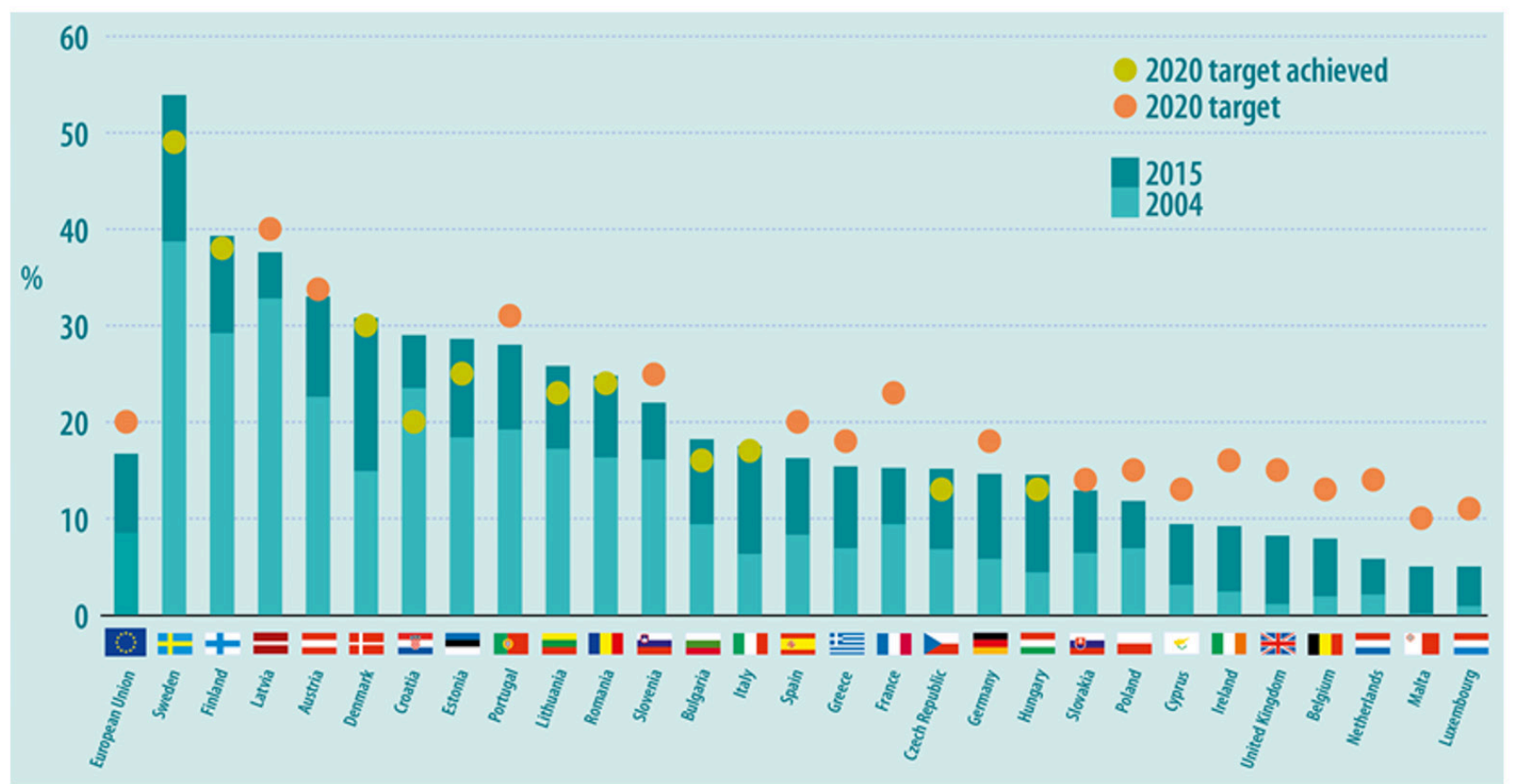

Fig. 1. Proporción de energía procedente de fuentes renovables en los Estados miembros de la UE en porcentaje del consumo energético final bruto. [1] 
El futuro del mercado energético plantea una nueva situación en la que una cantidad razonable de plantas de generación no es manejable, por lo que su suministro depende de factores externos, principalmente climáticos. Este es el caso de las dos tecnologías con mayor crecimiento en los últimos años: los parques eólicos y los campos solares, cuya producción depende de la intensidad del viento y de la irradiación solar respectivamente. Actualmente, las redes eléctricas gestionan la ratio generación - consumo mediante la regulación de la producción de las centrales para adecuarlo a la curva de demanda. Sin embargo, si la participación renovable continúa aumentando (Fig. 1.), la capacidad de gestión de los reguladores de la red disminuye hasta volverse insuficiente.

La industria de generación de energía se ve en gran medida influenciada por el incremento significativo de plantas de energía renovables en los últimos años y la creciente demanda de energía en el mundo, especialmente la electricidad, sumado a las preocupaciones sobre el medio ambiente requieren fuentes de energía más ecológicas y sostenibles, así como sistemas de conversión de energía con mayor eficiencia [2].

Las energías renovables tienen un papel importante en la generación de energía eléctrica y unas de las más desarrolladas actualmente (energía solar fotovoltaica y eólica) tienen la limitación de la intermitencia, lo que conduce a sistemas de respaldo con combustibles fósiles. Uno de los mayores desafíos que enfrenta el mundo en este momento es poder sustituir progresivamente el uso de energía fósil por energías más limpias en las plantas de generación de energía eléctrica.

El problema de la intermitencia se puede afrontar mediante el uso de sistemas de almacenamiento de energía en las plantas, siendo el almacenamiento de energía solar térmica (TES), utilizado en las plantas de energía solar concentrada (CSP), una de las tecnologías más prometedoras. Estas plantas enfrentan varios desafíos, incluidos los altos costos de generación actuales, pero las ventajas de los TES los convierten en una alternativa competente en un escenario energético futuro que evita el calentamiento global. De esta manera, dentro de las nuevas investigaciones existe una Hoja de Ruta de Demostración de Energía Solar Gen3 del Laboratorio Nacional de Energías Renovables (NREL) [3] que se ha centrado en la tecnología de Torre Central (SPT) con tres posibles caminos para el sistema de almacenamiento, el primero y más difundido, el de sales fundidas, y los que se encuentran en estudios son los de partículas y la fase gaseosa, donde el mayor desafío es no sobrepasar los costes fijados (Fig. 2.). Para todas estas vías, la Hoja de Ruta propone el ciclo de potencia Brayton de $\mathrm{CO}_{2}$ supercrítico $\left(\mathrm{s}-\mathrm{CO}_{2}\right)$. 


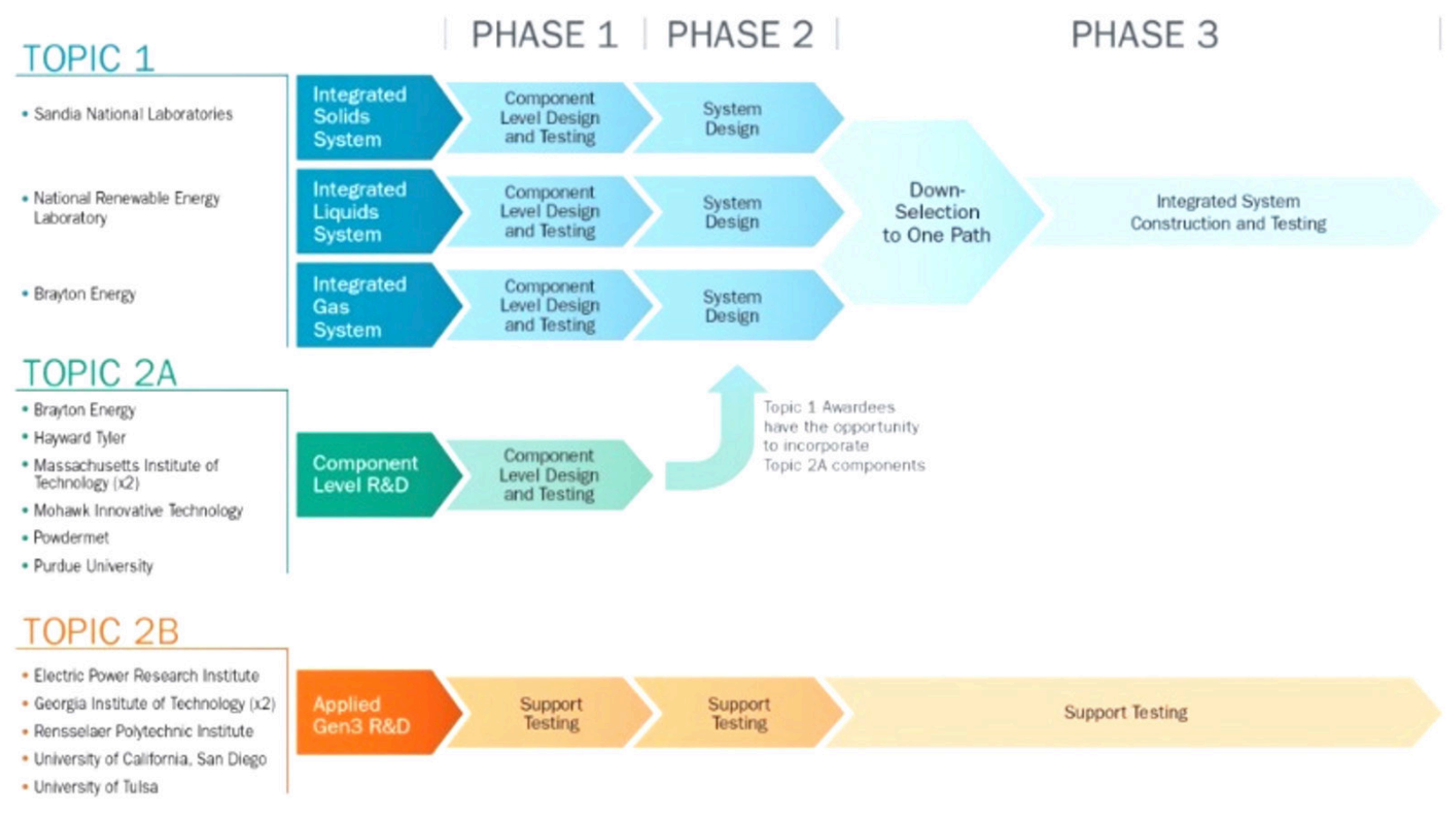

Fig. 2. Programa de investigación de CSP Gen3. Departamento de Energía de EE.UU. (DOE).

La concentración de energía solar típica aplicada en plantas de generación de energía enfoca el flujo solar diluido en un área pequeña a través del espejo óptico de diseño sofisticado. El área pequeña se conoce como un receptor donde el fluido de transferencia de calor (HTF) absorbe la radiación y finalmente transfiere la energía al vapor.

La CSP se distingue por poder entregar energía en participación de un almacenamiento de energía térmica (TES) de forma rentable y se caracteriza por la versatilidad en capacidad de salida. Su capacidad acumulativa global real y proyectada ha estado ganando impulso en los últimos años, siendo así que, se requieren $20 \mathrm{GWe}$ de nueva capacidad por año a nivel mundial hasta 2050 para satisfacer los objetivos internacionales de política energética según los expertos de la EIA [4]. Con el apoyo adecuado, para 2050, CSP podría proporcionar 9.6\% de la electricidad global solo de energía solar.

Es por ello que se han construido plantas de energía solar de gran capacidad como lo indica un informe del NREL, donde se menciona como ejemplos, el Proyecto de Energía Solar Crescent Dunes con una potencia de 110 MW y El Sistema de Generación Eléctrica Solar Ivanpah con una potencia de 392 MW en EE.UU., El Proyecto Solar Supcon con una potencia de 50MW en China y Proyecto Noor III de 150 MW de potencia en Marruecos [5].

Sin embargo, las plantas de energía solar que se han construido o que están en vía de desarrollo son principalmente impulsadas por ciclos de vapor sobrecalentado o vapor saturado (Rankine) con una eficiencia térmica relativamente baja y diseños complejos que impiden la electricidad solar tenga una reducción de LCOE (Levelized Cost of Energy) o costo ponderado de energía. En otras palabras, estas plantas de última generación no pueden competir con las que usan combustibles fósiles convencionales en términos de costes [6]. 
Los ciclos de potencia Brayton de $\mathrm{CO}_{2}$ supercrítico $\left(\mathrm{s}-\mathrm{CO}_{2}\right)$ han sido considerados ampliamente como una alternativa prometedora para la generación de energía debido a su alta eficiencia, diseño simple, compacidad y economía, también que operan con niveles de temperatura intermedios: energía solar concentrada (CSP) $[7,8]$, recuperación de calor residual [9,10], centrales eléctricas de carbón [11-12] y reactores nucleares Gen IV [13-15] entre otros.

En comparación con el ciclo Rankine, el ciclo Brayton de $\mathrm{s}-\mathrm{CO}_{2}$ permite lograr mejores eficiencias a temperaturas más bajas y presenta menores costos operativos y de capital ya que el $\mathrm{s}-\mathrm{CO}_{2}$ es varias veces más denso que el vapor. Esta característica permite operar con componentes compactos y simples que también requerirían menos mantenimiento. Además, térmicamente se adapta de mejor manera a las variaciones de temperatura del fluido de trabajo y su fuente de calor y, a su vez, logra una mayor eficiencia de la segunda ley (exergía), es decir, una mejor utilización del calor.

Por estas razones, se están analizando varias configuraciones simplificadas o más complejas de los ciclos de potencia de s- $\mathrm{CO}_{2}$ para la aplicación de conversión de calor a potencia de alto grado [16]. En general, existen principalmente dos métodos para mejorar la eficiencia del ciclo Brayton s- $\mathrm{CO}_{2}$. Uno de ellos es proponer el diseño de nuevos ciclos novedosos y se han investigado exhaustivamente en un reactor nuclear y una planta de energía solar térmica. Otro es usar una mezcla binaria adecuada basada en s- $\mathrm{CO}_{2}$ como fluido de trabajo en lugar de s- $\mathrm{CO}_{2}$ puro que se aplica principalmente en las centrales nucleares. La capacidad de conseguir una alta eficiencia en diversas aplicaciones con valores intermedios de temperatura hace que este ciclo se vuelva muy interesante y se proponga como objeto de estudio. La idea de combinar ciclos de potencia de $\mathrm{s}-\mathrm{CO}_{2}$ con CSP se ha identificado teóricamente viable. Se estima que se logra una reducción del $8 \%$ de LCOE al reemplazar el ciclo de vapor - Rankine con un bloque de potencia de $\mathrm{s}-\mathrm{CO}_{2}$ en plantas de torre central con sales fundidas como fluido de trabajo ya existente [17 - 19].

El trabajo realizado por Al-Sulaiman et al. (2015), determina que el ciclo de recompresión muestra un mejor rendimiento en comparación con otras configuraciones: simple, precompresión y expansión dividida. Mientras que, en el estudio de Marchionni et al. (2018), éste analiza ocho variantes del ciclo de potencia $\mathrm{s}-\mathrm{CO}_{2}$. Las métricas de energía, proceso y costo para diferentes diseños de ciclo se comparan al variar la temperatura a la entrada de la turbina (TIT) en el rango entre $250^{\circ} \mathrm{C}$ y $600^{\circ} \mathrm{C}$. El análisis realizado demuestra que las configuraciones del ciclo Brayton de s- $\mathrm{CO}_{2}$ más complejas conducen a una mayor eficiencia general y a una mayor producción neta de potencia, pero también a mayores costos de inversión (Fig. 3). Por el contrario, las arquitecturas más básicas, como el ciclo regenerativo simple, con un TIT de $425{ }^{\circ} \mathrm{C}$, podrían lograr una eficiencia general del aproximadamente un $25 \%$, una potencia de salida de alrededor de $94 \mathrm{kWe}$ y un período de recuperación bajo, menor a dos años. [16] 


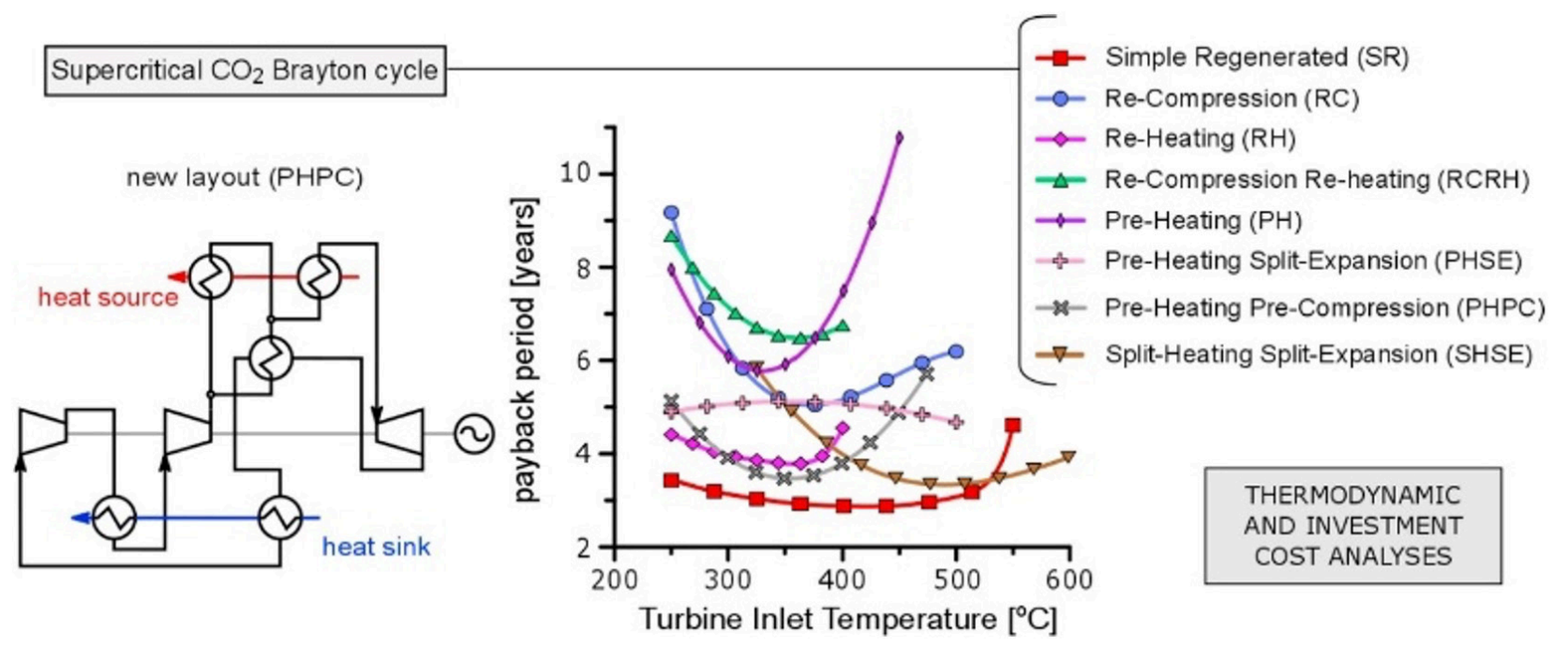

Fig. 3. Configuraciones de Ciclos de Potencia s-CO2. PayBack vs. Temperatura de entrada a la turbina.

Neises, T., y Turchi, C. (2019), realizan un estudio del diseño, rendimiento y coste de las configuraciones simples de recompresión y de enfriamiento parcial del ciclo de potencia de dióxido de carbono supercrítico integrado con una Torre Central de sales fundidas como fluido de transferencia de calor. En dicho estudio utilizan un modelo de estado estable para diseñar cada ciclo con cantidades variables de conductancia del recuperador para comprender el rendimiento y las compensaciones de costos. Donde se concluye que, el LCOE mínimo del ciclo de enfriamiento parcial es aproximadamente $6.2 \%$ menor que el LCOE mínimo del ciclo simple de recompresión. Existen algunas ventajas con el ciclo con enfriamiento parcial. La más importante es que al tener una diferencia de temperatura más grande en el intercambiador de calor primario (PHX) hace que se tenga diferencial de temperatura más grande en TES, lo que favorece al sistema de almacenamiento de energía porque se tendría un menor coste, y, por tanto, un menor coste en la instalación. Es conveniente tener una temperatura de trabajo más fría en ciclo de enfriamiento parcial ya que se ha comprobado que se minimizan las pérdidas térmicas. Finalmente, cuando mayor sea la diferencia de temperatura, se va a tener un menor caudal másico del HTF, lo que nos llevaría a un menor consumo de electricidad en la bomba.

En el estudio de [18], proponen varias configuraciones nuevas de ciclo de s-CO2 Brayton con recompresión para plantas solares de torre central (Fig. 4.). En donde el ciclo propuesto se suministra la energía térmica por del lado de baja presión con parámetros de 85 bar en la entrada principal de calor y menos de 200 bar en la entrada del recalentamiento, lo que permite utilizar los intercambiadores de calor de tipo carcasa y tubos donde circula la sal fundida. En los dos escenarios que propone, enfriamiento seco y enfriamiento húmedo, demuestra que, en el primero, se logra la mayor eficiencia en la recompresión con diseño de enfriamiento y recalentamiento, alcanzando un valor de $52.6 \%$ a 300 bar de presión de entrada de la turbina. Mientras que, en el escenario de enfriamiento húmedo, el ciclo de recompresión sin intercooler y no recalentado supera el $51 \%$ de eficiencia a 250 bar, aumentando a más del $54 \%$ si se agrega el recalentamiento. Además, concluye que, si la eficiencia del ciclo al $50 \%$ se establece como un objetivo para las plantas solares avanzadas, la recompresión en el escenario de enfriamiento húmedo podría ser considerado como el primer prototipo en probar en esta nueva tecnología. 


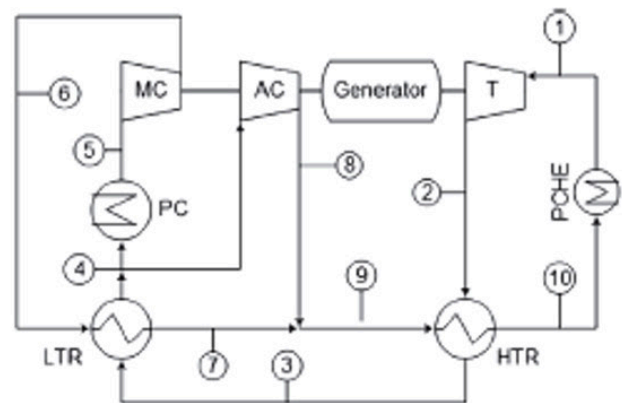

(a) Conventional re-compressed layout ( $\mathrm{RC}$ )

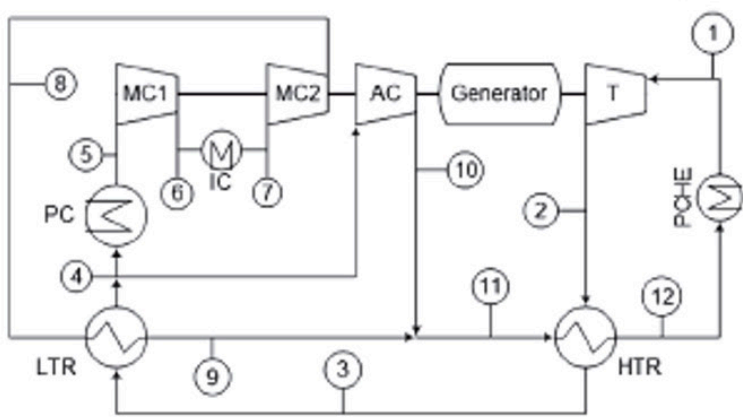

(c) Conventional re-compressed with intercooling layout (RC-IC)

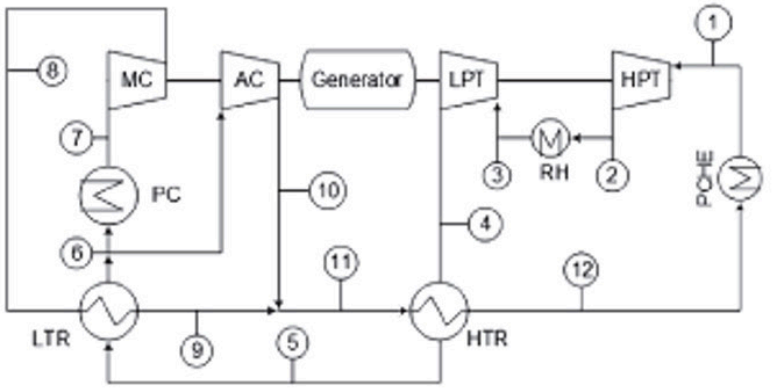

(e) Conventional re-compressed with reheating layout (RC-RH)

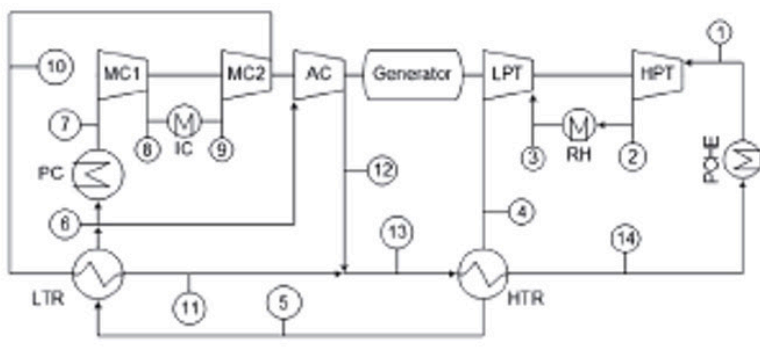

(g) Conventional re-compressed with intercooling and reheating layout (RC-IC-RH)

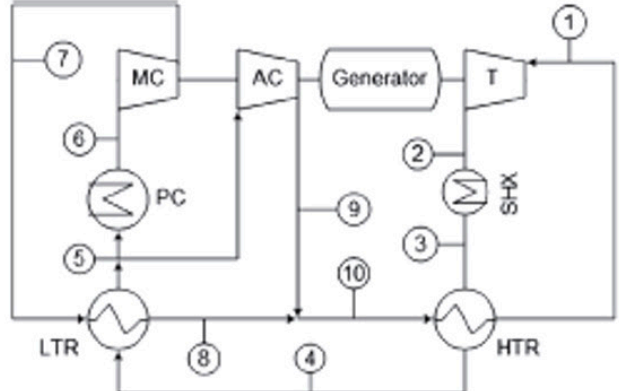

(b) Proposed re-compressed layout (RC-LP)

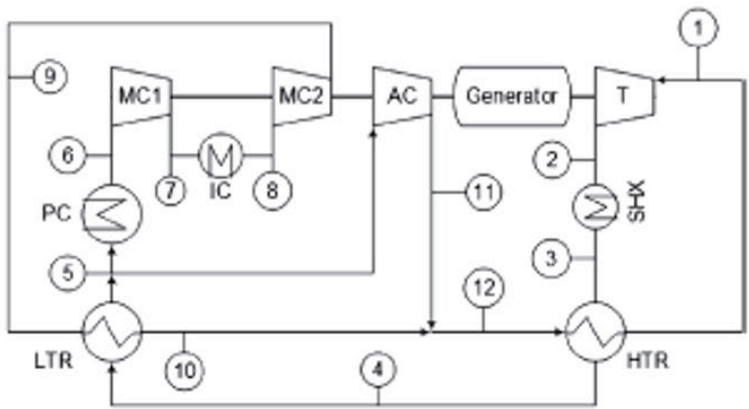

(d) Proposed re-compressed with intercooling layout (RC-IC-LP)

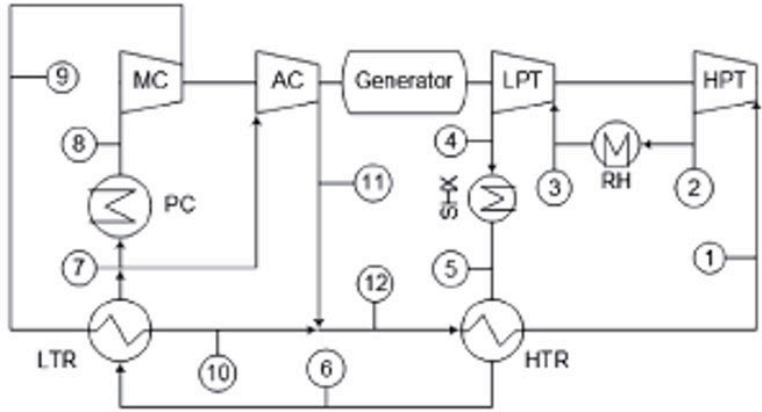

(f) Proposed re-compressed with reheating layout (RC-RH-LP)

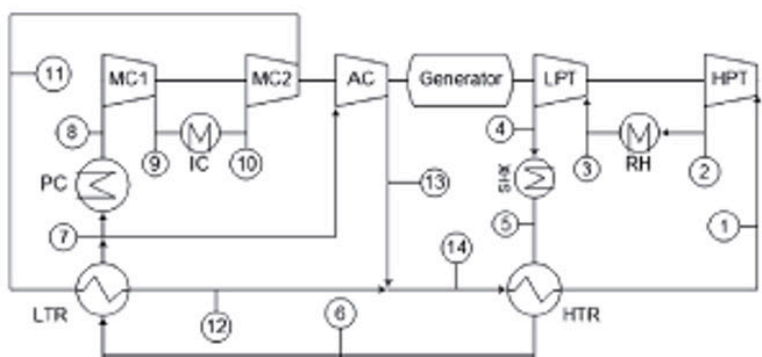

(h) Proposed re-compressed with intercooling and reheating layout (RC-IC-RH-LP)

Fig. 4. Diseños analizados $a, c, e, g$ convencionales y $b, d, f$, $h$ propuestos [18]

Posteriormente afirma que los ciclos propuestos, (Fig. 4.) tienden a aprovechar una presión de entrada de la turbina más alta con lo cual logran aumentar el trabajo del ciclo específico, compensando así la temperatura de entrada de la turbina más baja. Y que, además, la reducción en la presión de trabajo de los intercambiadores de calor utilizados en el estudio permiten disminuir significativamente el coste logrando una mayor compacidad, es decir, una mejor respuesta dinámica y menores inversiones, manteniendo la eficiencia [18]. 
Finalmente, [19], estudia la influencia de las mezclas de fluidos a base de dióxido de carbono en un ciclo Brayton s- $\mathrm{CO}_{2}$ con una configuración de recompresión, este ciclo es la evolución de una configuración anterior propuesta por Angelino et al. (1968), que también fueron estudiadas por Crespi et al. (2017) y Coco - Enríquez (2017). En la investigación [19] los autores señalan varias conclusiones, primero, existen dos grupos A y B: "A" son mezclas que disminuyen la temperatura crítica, en las que se incluye s- $\mathrm{CO}_{2} / \mathrm{He}, \mathrm{s}-\mathrm{CO}_{2} / \mathrm{Kr}$, $\mathrm{s}-\mathrm{CO}_{2} / \mathrm{CH}_{4}$ y s- $\mathrm{CO}_{2} / \mathrm{C}_{2} \mathrm{H}_{6}$ y " $\mathrm{B}$ " son mezclas que aumentan la temperatura crítica, s- $\mathrm{CO}_{2} /$ $\mathrm{H}_{2} \mathrm{~S}, \mathrm{~s}-\mathrm{CO}_{2} / \mathrm{C}_{3} \mathrm{H}_{8}, \mathrm{~s}-\mathrm{CO}_{2} / \mathrm{C}_{4} \mathrm{H}_{10}, \mathrm{~s}-\mathrm{CO}_{2} / \mathrm{C}_{5} \mathrm{H}_{10}, \mathrm{~s}-\mathrm{CO}_{2} / \mathrm{C}_{5} \mathrm{H}_{12}$, entre otros. $\mathrm{Y}$, segundo, que en el caso de las mezclas de grupo A la eficiencia del ciclo es máxima cuando el ciclo funciona con una temperatura a la entrada del compresor (CIT) y presión a la entrada del compresor $(\mathrm{CIP})$ cercano al punto crítico. Cuando se realiza la comparación entre el ciclo con s- $\mathrm{CO}_{2}$ puro y usando mezclas del grupo $A$, se observa que en este último las eficiencias aumentan aproximadamente un $4 \%$.

Mientras que si se compara el ciclo con s- $\mathrm{CO}_{2}$ puro y las mezclas del grupo $\mathrm{B}$, se puede observar que la eficiencia del ciclo disminuye cuando funciona con CIT y CIP justo por encima del punto crítico. Sin embargo, la eficiencia del ciclo solamente mejora en aproximadamente 3 a 4\%, cuando el CIP se encuentra justo por encima de la presión crítica y $\mathrm{CIT}=318.15 \mathrm{~K}$.

Como indica la literatura, se han analizado diferentes configuraciones de ciclos de potencia para CSP, además se conoce que tanto las mezclas que disminuyen como las que aumentan la temperatura crítica incrementan la eficiencia del ciclo de potencia Brayton $\mathrm{s}-\mathrm{CO}_{2}$. Siguiendo esta tendencia, es importante seguir realizando estudios de las diferentes mezclas a base de s- $\mathrm{CO}_{2}$ en varios ciclos de potencia Brayton, lo cual nos conducirá a poder encontrar una o varias substancias y una o varias configuraciones que maximicen la eficiencia, que reduzcan costes de generación de energía eléctrica y que, además, puedan ser aplicadas a la industria de las plantas de CSP.

\section{Fundamento Teórico}

El software denominado SCSP (Planta de Energía Solar Concentrada Supercrítica) se puede considerar como un potente simulador del rendimiento de distintas configuraciones simples y complejas de ciclos de potencia Brayton utilizando mezclas binarias, ternarias y cuaternarias a base de dióxido de carbono y el fluido estándar ( $\mathrm{s}-\mathrm{CO}_{2}$ puro). Este programa ha sido desarrollado por miembros del Departamento de Ingeniería Energética de la Universidad Politécnica de Madrid [17] y su núcleo se basa en el software desarrollado por J. Dyreby (2014), en su tesis doctoral: Modeling the supercritical carbon dioxide Brayton cycle with recompression [21]. Este programa tiene desarrollado un código Fortran donde se incluye configuraciones innovadoras que cuentan con recalentamientos en las etapas intermedias de expansión en la turbina. Se incluye también el dimensionamiento en detalle de los recuperadores y los intercambiadores de calor. Estas configuraciones de ciclos Brayton están acopladas a modelos de campo solar con colectores lineales Cilindro Parabólicos (PTC) y Lineales de Fresnel (LF), y con diferentes fluidos de transferencia de calor (HTF) como sales fundidas, aceites térmicos y DSG. SCSP establece una conductancia total del recuperador de calor constante como metodología de cálculo del rendimiento y utiliza algoritmos para la optimización de los parámetros de operación del ciclo (SUBPLEX, NEWUOA y BOBYQA) con el objetivo de maximizar el rendimiento térmico del ciclo. Una vez establecido el valor de la conductancia se pueden variar parámetros importantes 
compresor y la turbina, presión a la entrada del compresor y turbina, presiones intermedias y de recalentamiento, eficiencias isentrópicas de los compresor y turbinas, y, además las caídas de presión en los diferentes componentes del ciclo. Las propiedades de los fluidos se obtienen de la Base de datos de propiedades de transporte y termodinámica de fluidos de referencia (REFPROP) desarrollada por NIST en los EE. UU. [21]. La Fig. 5 muestra el proceso de iteración para los modelos de los ciclos integrados en el software SCSP.

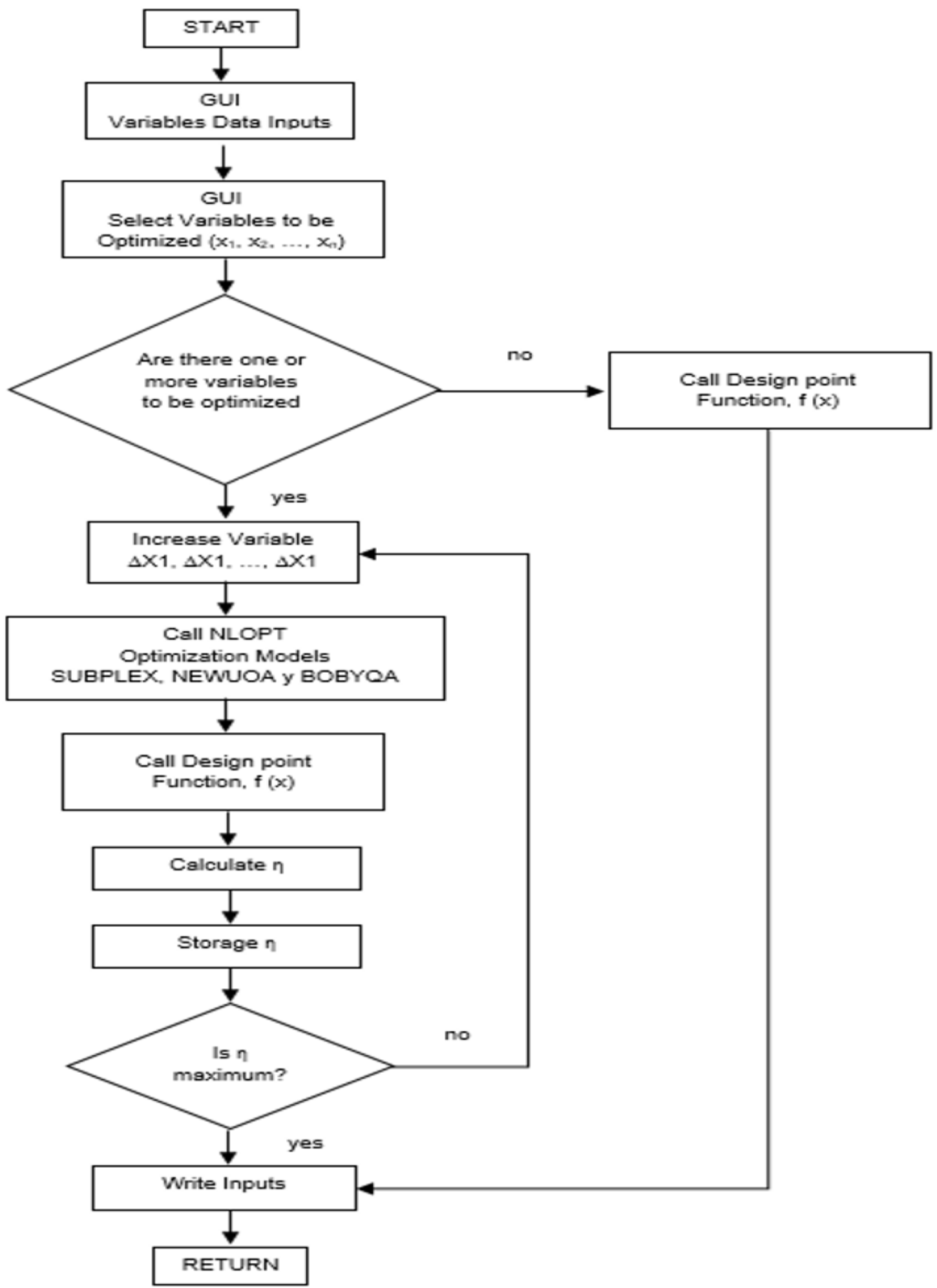

Fig. 5. Proceso de iteración para los modelos de ciclos integrados en el software SCSP. [19] 


\section{Resultados y Validación del Software SCSP}

Para validar los diferentes ciclos que tiene configurado este simulador SCSP se utiliza el software Thermoflex [22], en el cual se modela cada una de las configuraciones de ciclos de potencia Brayton. Como ejemplos en este caso se presenta la validación de las configuraciones de Recompresión con dos recalentamientos y Recompresión con enfriamiento intermedio en el compresor principal y con dos recalentamientos.

\section{Ciclo de Recompresión}

Una vez modelado el ciclo en Thermoflex se obtienen los datos de temperatura, presión, entalpía y entropía en cada punto (Fig. 6.), para validar este último dato se toma en cuenta los valores de los dos primeros, temperatura $(T)$ y presión $(P)$, y mediante la función: $h=$ Enthalpy (Carbon Dioxide, $T=T, P=P$ ) del software EES se obtiene las entalpías.

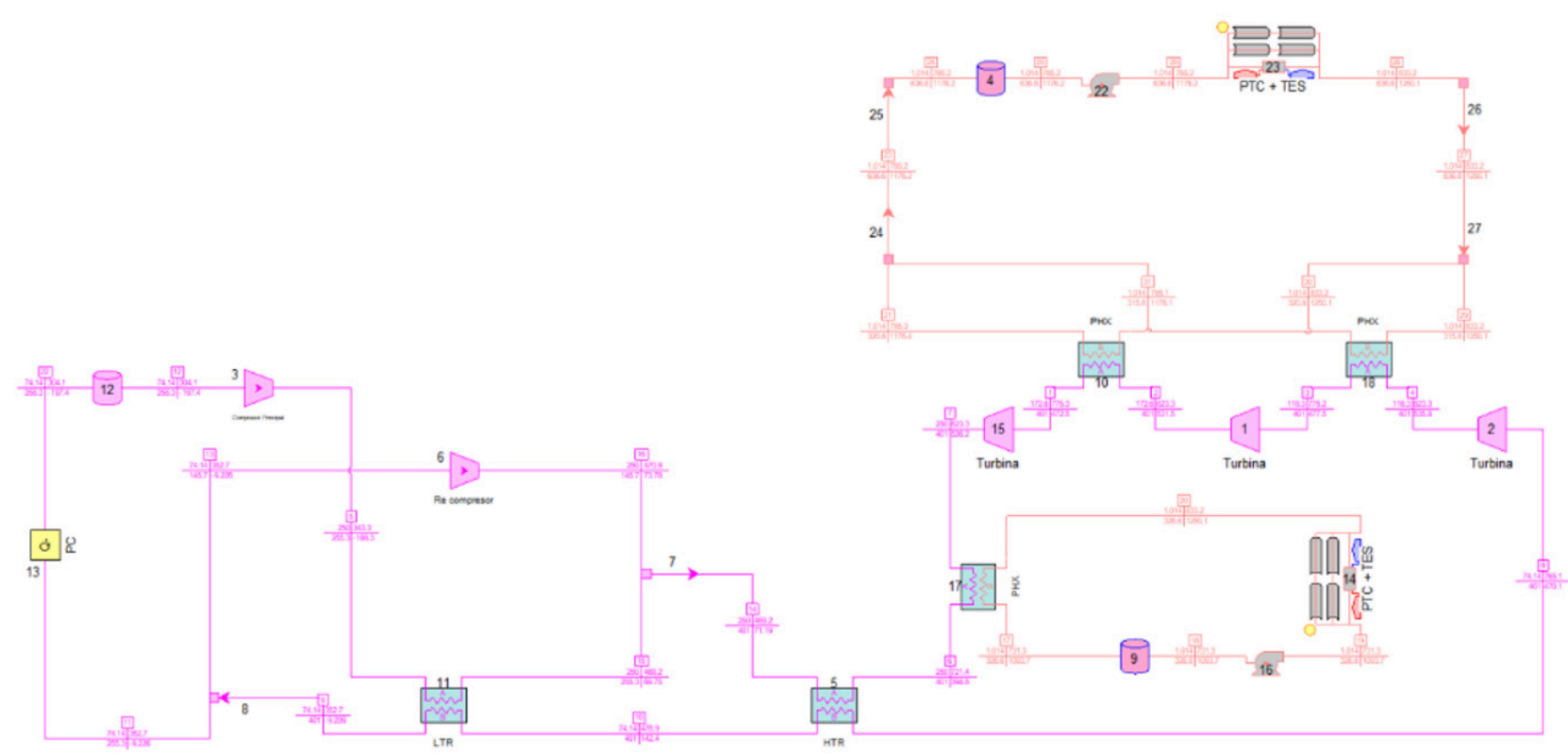

Fig. 6. Esquema de la modelación del ciclo RCC con dos recalentamientos (UA 1OMW/K) acoplado con CSP y TES

Por tanto, para validar todos los datos se obtiene el rendimiento térmico del ciclo (RCC $2 \mathrm{RH}$ ) utilizando la siguiente formulación:

$$
\begin{gathered}
\eta_{\text {th }}=\frac{\dot{\mathrm{W}}_{\text {Realizado }}}{\dot{\mathrm{Q}}_{\text {Aportado }}} \\
\eta_{\mathrm{th}}=\frac{\dot{\mathrm{W}}_{\mathrm{T}}-\dot{\mathrm{W}}_{\mathrm{MC}}-\dot{\mathrm{W}}_{\mathrm{RC}}}{\dot{\mathrm{Q}}_{\mathrm{PHX}}+\dot{\mathrm{Q}}_{\mathrm{RHX} 1}+\dot{\mathrm{Q}}_{\mathrm{RHX} 2}}
\end{gathered}
$$




$$
\eta_{\text {th }}=\frac{\left(\frac{\dot{\mathrm{m}}_{\mathrm{T}}}{\dot{\mathrm{m}}_{\mathrm{T}}} * \mathrm{~W}_{\mathrm{T}}-\frac{\dot{\mathrm{m}}_{\mathrm{MC}}}{\dot{\mathrm{m}}_{\mathrm{T}}} * \mathrm{~W}_{\mathrm{MC}}-\frac{\dot{\mathrm{m}}_{\mathrm{RC}}}{\dot{\mathrm{m}}_{\mathrm{T}}} * \mathrm{~W}_{\mathrm{RC}}\right)}{\frac{\dot{\mathrm{Q}}_{\mathrm{PHX}}}{\dot{\mathrm{m}}_{\mathrm{T}}}+\frac{\dot{\mathrm{Q}}_{\mathrm{RHX} 1}}{\dot{\mathrm{m}}_{\mathrm{T}}}+\frac{\dot{\mathrm{Q}}_{\mathrm{RHX} 2}}{\dot{\mathrm{m}}_{\mathrm{T}}}}
$$

$$
\eta_{\mathrm{th}}=\frac{\mathrm{W}_{\mathrm{T} 1}+\mathrm{W}_{\mathrm{T} 2}+\mathrm{W}_{\mathrm{T} 3}-(1-\gamma) * \mathrm{~W}_{\mathrm{MC}}-\gamma * \mathrm{~W}_{\mathrm{RC}}}{\mathrm{Q}_{\mathrm{PHX}}+\mathrm{Q}_{\mathrm{RHX} 1}+\mathrm{Q}_{\mathrm{RHX} 2}}
$$

$$
\eta_{\text {th }}=\frac{\left(h_{6}-h_{11}\right)+\left(h_{6}-h_{11}\right)+\left(h_{14}-h_{7}\right)-(1-\gamma) *\left(h_{2}-h_{1}\right)-\gamma *\left(h_{10}-h_{9}\right)}{\left(h_{6}-h_{5}\right)+\left(h_{12}-h_{11}\right)+\left(h_{14}-h_{13}\right)}
$$

Donde $\eta_{\text {th }}$ es el rendimiento térmico del ciclo, $\hat{W}_{\text {Realizado }}$ es el trabajo neto realizado por el ciclo, $\mathbb{Q}_{\text {Aportado }}$ es el calor aportado hacia el ciclo, $\hat{W}_{T}, W_{M C}, \hat{W}_{\mathrm{RC}}$ son los trabajos de la turbina, compresor y recompresor respectivamente, $\mathrm{Q}_{\mathrm{PHX}} \mathrm{Q}_{\mathrm{RHX} 1} \mathrm{Q}_{\mathrm{RHX} 2}$ son los calores aportados por el intercambiador de calor primario e intercambiadores de calor de recalentamiento, $\mathrm{m}_{\mathrm{T}}$ es el flujo másico total que circula por el ciclo, $m_{M C}$ y $m_{R C}$ son los flujos másicos que se desvían al compresor principal y al recompresor respetivamente y $h_{1}$ a $h_{14}$ son las entalpías en cada uno de los puntos del ciclo. Siendo y la fracción de mezcla que se desvía hacia recompresor con un valor de 0.3633 . Con lo cual se obtuvo los siguientes resultados:

Tabla 1. Valores de eficiencia con los diferentes software

\begin{tabular}{|c|c|c|c|c|}
\hline Software & SCSP & $\begin{array}{c}\text { Thermoflex } \\
\text { (Ecuación) }\end{array}$ & EES (Ecuación) & $\begin{array}{c}\text { Error } \\
\text { (SCSP - EES) }\end{array}$ \\
\hline$\eta_{\mathrm{th}}$ & $51.0822 \%$ & $51.1341 \%$ & $51.0788 \%$ & $0.0066 \%$ \\
\hline
\end{tabular}

Los valores obtenidos de la eficiencia térmica mostrados (Tabla 1.) demuestran que las configuraciones realizadas en el software SCSP (con $\eta_{\text {th }}=51.0822 \%$ ) quedan completamente validadas para realizar diferentes simulaciones con fluidos supercríticos como fluido de trabajo.

\section{Ciclo de Recompresión con enfriamiento intermedio en el compresor principal}

Ocupando el criterio anterior, se realiza el modelado del ciclo $\mathrm{RCMCl}$ - RH (Fig. 7.) y se realiza el análisis propuesto en el apartado anterior para obtener los valores de eficiencia. 


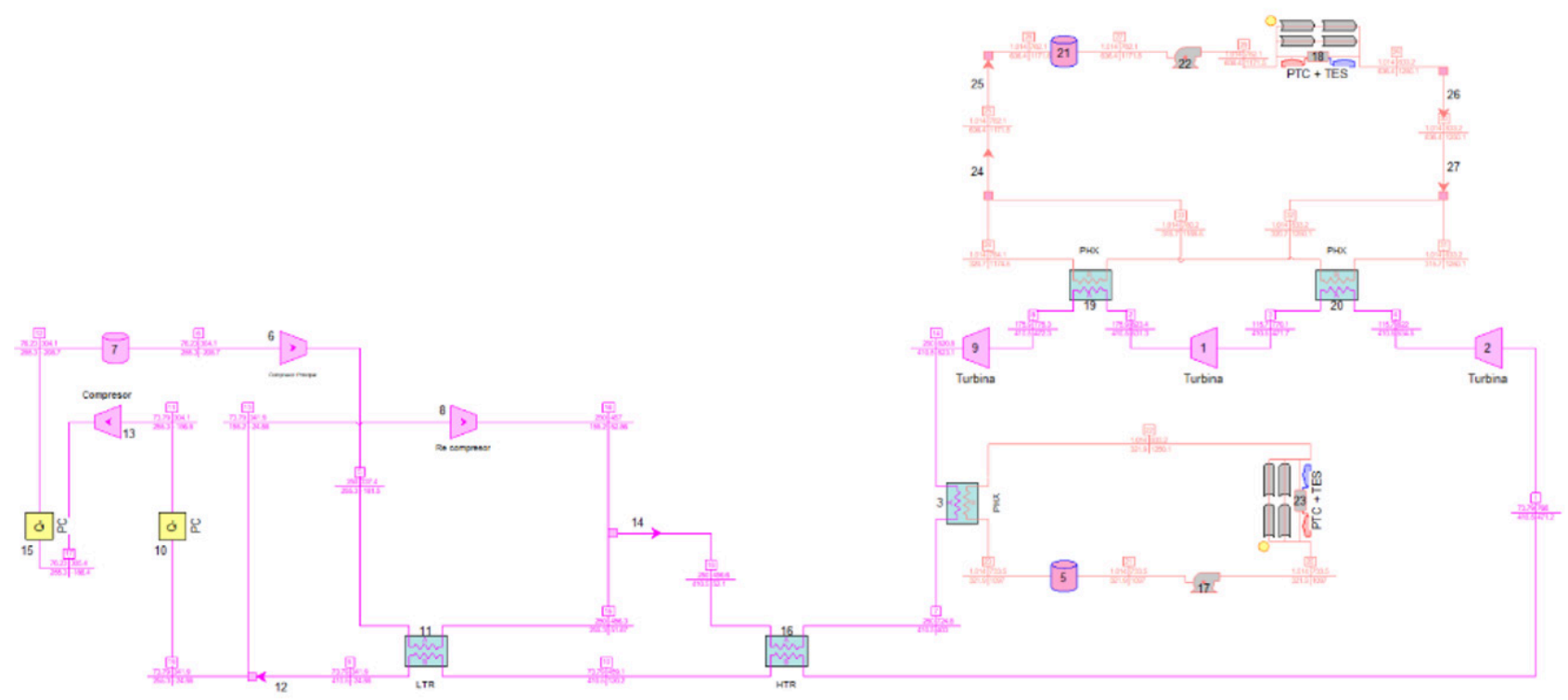

Fig. 7. Esquema de la modelación del ciclo $R C M C l$ con dos recalentamientos (UA $10 M W / K$ ) acoplado con CSP y TES

Del mismo modo, para validar todos los datos se obtiene el rendimiento térmico del ciclo (RCC - 2RH) utilizando la siguiente formulación:

$$
\begin{gathered}
\eta_{\text {th }}=\frac{\dot{\mathrm{W}}_{\text {Realizado }}}{\dot{\mathrm{Q}}_{\text {Aportado }}} \\
\eta_{\mathrm{th}}=\frac{\dot{\mathrm{W}}_{\mathrm{T}}-\dot{\mathrm{W}}_{\mathrm{C}}-\dot{\mathrm{W}}_{\mathrm{MC}}-\dot{\mathrm{W}}_{\mathrm{RC}}}{\dot{\mathrm{Q}}_{\mathrm{PHX}}+\dot{\mathrm{Q}}_{\mathrm{RHX} 1}+\dot{\mathrm{Q}}_{\mathrm{RHX} 2}}(7) \\
\eta_{\mathrm{th}}=\frac{\left(\frac{\dot{\mathrm{m}}_{\mathrm{T}}}{\dot{\mathrm{m}}_{\mathrm{T}}} \mathrm{W}_{\mathrm{T}}-\frac{\left.\dot{\mathrm{m}}_{\mathrm{C}} * \mathrm{~W}_{\mathrm{C}}-\frac{\dot{\mathrm{m}}_{\mathrm{MC}}}{\dot{\mathrm{m}}_{\mathrm{T}}} * \mathrm{~W}_{\mathrm{MC}}-\frac{\dot{\mathrm{m}}_{\mathrm{RC}}}{\dot{\mathrm{m}}_{\mathrm{T}}} * \mathrm{~W}_{\mathrm{RC}}\right)}{\frac{\dot{\mathrm{Q}}_{\mathrm{PHX}}}{\dot{\mathrm{m}}_{\mathrm{T}}}+\frac{\dot{\mathrm{Q}}_{\mathrm{RHX} 1}}{\dot{\mathrm{m}}_{\mathrm{T}}}+\frac{\dot{\mathrm{Q}}_{\mathrm{RHX} 2}}{\dot{\mathrm{m}}_{\mathrm{T}}}}\right.}{\eta_{\mathrm{th}}=\frac{\mathrm{W}_{\mathrm{T} 1}+\mathrm{W}_{\mathrm{T} 2}+\mathrm{W}_{\mathrm{T} 3}-(1-\gamma) * \mathrm{~W}_{\mathrm{C}}-(1-\gamma) * \mathrm{~W}_{\mathrm{MC}}-\gamma * \mathrm{~W}_{\mathrm{RC}}}{\mathrm{Q}_{\mathrm{PHX}}+\mathrm{Q}_{\mathrm{RHX} 1}+\mathrm{Q}_{\mathrm{RHX} 2}}}
\end{gathered}
$$

$$
\eta_{\text {th }}=\frac{\left(h_{6}-h_{11}\right)+\left(h_{12}-h_{15}\right)+\left(h_{16}-h_{7}\right)-(1-\gamma) *\left(h_{14}-h_{13}\right)-(1-\gamma) *\left(h_{2}-h_{1}\right)-\gamma *\left(h_{10}-h_{9}\right)}{\left(h_{6}-h_{5}\right)+\left(h_{12}-h_{11}\right)+\left(h_{16}-h_{15}\right)}
$$


Siendo y la fracción de mezcla que se desvía hacia recompresor con un valor de 0.3776 . Con lo cual se obtuvo los siguientes resultados:

Tabla 2. Valores de eficiencia con los diferentes software.

\begin{tabular}{|c|c|c|c|c|}
\hline Software & SCSP & $\begin{array}{c}\text { Thermoflex } \\
\text { (Ecuación) }\end{array}$ & $\begin{array}{c}\text { EES } \\
\text { (Ecuación) }\end{array}$ & $\begin{array}{c}\text { Error } \\
\text { (SCSP - EES) }\end{array}$ \\
\hline$\eta_{\text {th }}$ & $51.2484 \%$ & $51.1627 \%$ & $51.2351 \%$ & $0.0260 \%$ \\
\hline
\end{tabular}

De la misma manera, los valores obtenidos de la eficiencia térmica mostrados (Tabla 2.) demuestran que las configuraciones realizadas en el software SCSP (con $\eta_{\text {th }}=51.2484$ \%) quedan completamente validadas para realizar diferentes simulaciones con fluidos supercríticos como fluido de trabajo.

Por tanto, el estudio, análisis y simulación de la eficiencia térmica en los ciclos Brayton usando $\mathrm{CO}_{2}$ supercrítico y mezclas binarias a base del fluido estándar $\left(\mathrm{s}-\mathrm{CO}_{2}\right)$ como fluidos de trabajo se puede realizar ocupando el software SCSP. Dentro de las simulaciones en las diferentes configuraciones de ciclos de potencia se pueden considerar varios parámetros importantes para el análisis de la variación de eficiencia térmica como por ejemplo: la temperatura de entrada al compresor, presión a la entrada del compresor, temperatura de entrada a la turbina, presión a la entrada de la turbina, presión intermedia y a la entrada del recalentador, conductancia de los recuperadores de calor de baja y alta temperatura, fracción que se dirige al recompresor, caídas de presión en todos los componentes del ciclo y las eficiencias isentrópicas de la turbinas, compresor y recompresor.

Los usos de las mezclas deben logran mayores eficiencias para permitir una reducción drástica en el tamaño de la planta termosolar y, por lo tanto, en el costo del campo solar, que es el principal contribuyente a los costos de capital. También necesitan temperaturas de funcionamiento más altas para producir una reducción drástica del tamaño de la planta $y$, por tanto, del coste del sistema de almacenamiento. En este sentido el estudio de las mezclas a base de dióxido de carbono supercrítico se vuelve fundamental debido a que sus propiedades termofísicas y termoquímicas como la capacidad calorífica, viscosidad cinemática, conductividad térmica, nusselt, Reynolds, etc, permitirán una mejor comprensión de su comportamiento como fluido de trabajo en las diferentes configuraciones de ciclos de potencia.

\section{Conclusiones y trabajos futuros}

Como principal conclusión se indica que las variaciones de las propiedades del fluido de trabajo tienen una incidencia directa positiva en los ciclos de potencia Brayton de CO2 supercríticos, dentro de estos ciclos las configuraciones que se encuentran en estudio son las de Recompresión y Recompresión con enfriamiento intermedio en el compresor principal ya que obtienen mejor eficiencia que otras configuraciones dependiendo de los parámetros de entrada como: temperatura ambiente, temperatura de entrada al compresor, temperatura de entrada a la turbina y la presión máxima del ciclo, entre otras. 
Existen diferentes aspectos que se deben considerar al seleccionar una mezcla óptima, ya sea esta binaria, ternaria o cuaternaria. Las dimensiones de los componentes del ciclo se encuentran limitados por varios factores y uno de los principales es densidad del fluido de trabajo. Si la densidad aumenta, entonces los tamaños de los compresores y turbinas se minimizarán. Además, en el diseño de los Intercambiadores de Calor se deben tener en cuenta parámetros como: la conductividad térmica, la capacidad calorífica isobárica (Cp) y la viscosidad cinética. Se conoce que los coeficientes de transferencia de calor que se involucran en el sistema intercambiador de calor están relacionados con la viscosidad (dinámica y cinética), conductividad térmica, densidad y capacidad calorífica isobárica. "Los valores más altos de Cp y densidad; y valores más bajos de viscosidad cinemática logran minimizar las dimensiones de los recuperadores de calor de baja y alta temperatura (LTR y HTR)".

Como se mencionó anteriormente, existen mezclas cuyas propiedades pueden ayudar a redistribuir el punto crítico, es decir aumentar o disminuir la temperatura crítica en comparación con la temperatura crítica del fluido estándar. Dichas mezclas a base de $\mathrm{CO}_{2}$ supercrítico pueden llegar a aumentar la eficiencia en un 3 y $4 \%$ dependiendo tanto de la temperatura ambiente en las que se encuentren trabajando, la temperatura de entrada al compresor y la temperatura de entrada a la turbina. También es importante analizar las pérdidas de presión en los componentes del sistema ya que las irreversibilidades causadas por estos puede tener repercusiones negativas en la eficiencia de cada una de las configuraciones de ciclos de potencia, es por ello que la comunidad científica y en sí nuestro trabajo futuro tiene en consideración todos estos parámetros para ser analizados y apuesta por el estudio de estos fluidos de trabajo en ciclos Brayton que cuentan con diferentes configuraciones como simple, recompresión, recompresión con enfriamiento intermedio en el compresor principal y recompresión con enfriamiento parcial; y que se encuentran acoplados a plantas de energía solar concentrada.

\section{Referencias}

Euroheat \& Power. 2020. Share of Renewables in EU Energy Consumption Reached 17\% In 2016 | Euroheat \& Power. [online] Available at: <https://www.euroheat.org/news/ share-renewables-energy-consumption-eu-reached-17-2016/>. Accedido el 26 Marzo de 2021].

Löffler, K., Burandt, T., Hainsch, K., \& Oei, P. Y. Modeling the low-carbon transition of the European energy system-A quantitative assessment of the stranded assets problem. Energy Strategy Reviews, 26, 100422. (2019)

Mehos, M., Turchi, C., Vidal, J., Wagner, M., Ma, Z., Ho, C., ... \& Kruizenga, A. Concentrating solar power Gen3 demonstration roadmap (No. NREL/TP-5500-67464). National Renewable Energy Lab.(NREL), Golden, CO (United States). (2017). https://doi. org/10.2172/1338899

https://solarpaces.nrel.gov/.

Yin, J. M., Zheng, Q. Y., Peng, Z. R., \& Zhang, X. R. Review of supercritical CO2 power cycles integrated with CSP. International Journal of Energy Research. (2019). 
Zhu, H. H., Wang, K., \& He, Y. L. Thermodynamic analysis and comparison for different direct-heated supercritical CO2 Brayton cycles integrated into a solar thermal power tower system. Energy, 140, 144-157, (2017).

Ma, Z., \& Turchi, C. S. Advanced supercritical carbon dioxide power cycle configurations for use in concentrating solar power systems (No. NREL/CP-5500-50787). National Renewable Energy Lab.(NREL), Golden, CO (United States). (2011).

Manente, G., \& Lazzaretto, A. Innovative biomass to power conversion systems based on cascaded supercritical CO2 Brayton cycles. Biomass and Bioenergy, 69, 155-168, (2014).

Wu, C., Yin, X., Ma, L., Zhou, Z., \& Chen, H. Design and operation of a 5.5 MWe biomass integrated gasification combined cycle demonstration plant. Energy \& fuels, 22(6), 4259-4264, (2008).

Mecheri, M.; Le, Y. Supercritical CO2 Brayton Cycles for Coal- Fired Power Plants. Energy 2016, 103, 758-771. [CrossRef]

Park, S., Kim, J., Yoon, M., Rhim, D. , \& Yeom, C. Thermodynamic and economic investigation of coal-fired power plant combined with various supercritical CO2 Brayton power cycle. Applied Thermal Engineering, 130, 611-623, (2018).

Li, M. J., Zhu, H. H., Guo, J. Q., Wang, K., \& Tao, W. Q. The development technology and applications of supercritical CO2 power cycle in nuclear energy, solar energy and other energy industries. Applied Thermal Engineering, 126, 255-275, (2017).

Li, M. J., Jie, Y. J., Zhu, H. H., Qi, G. J., \& Li, M. J. The thermodynamic and cost-benefitanalysis of miniaturized lead-cooled fast reactor with supercritical $\mathrm{CO} 2$ power cycle in the commercial market. Progress in Nuclear Energy, 103, 135-150, (2018).

Qi, H., Gui, N., Yang, X., Tu, J., \& Jiang, S. The application of supercritical CO2 in nuclear engineering: A review. The Journal of Computational Multiphase Flows, 10(4), 149158, (2018).

Crespi, F., Gavagnin, G., Sánchez, D., \& Martínez, G. S. Supercritical carbon dioxide cycles for power generation: A review. Applied Energy, 195, 152-183, (2017).

Enriquez, L. C. (2017). Nueva generación de centrales termosolares con colectores solares lineales acoplados a ciclos supercríticos de potencia (Doctoral dissertation, Universidad Politécnica de Madrid).

Linares, J. I., Montes, M. J., Cantizano, A., \& Sánchez, C. A novel supercritical CO2 recompression Brayton power cycle for power tower concentrating solar plants. Applied Energy, 263, 114644, (2020).

Valencia-Chapi, R., Coco-Enríquez, L., \& Muñoz-Antón, J. Supercritical CO2 Mixtures for Advanced Brayton Power Cycles in Line-Focusing Solar Power Plants. Applied Sciences, 10(1), 55, (2020). 
E. W. Lemmon, I. H. Bell, M. L. Huber, and M. O. McLinden, "NIST Standard Reference Database 23: Reference Fluid Thermodynamic and Transport Properties-REFPROP, Version 10.0", 2018.

Dyreby, J. J. (2014). Modeling the supercritical carbon dioxide Brayton cycle with recompression (Doctoral dissertation, The University of Wisconsin-Madison).

Thermoflow Software manual. https://www.thermoflow.com/ 